\title{
Paediatric Early Warning Systems: Myths and Muses
}

Dr. Damian Roland

Consultant and Honorary Senior Lecturer in Paediatric Emergency Medicine

1. SAPPHIRE Group, Health Sciences, Leicester University, UK

2. Paediatric Emergency Medicine Leicester Academic (PEMLA) Group, Children's Emergency Department, Leicester Hospitals, UK

e-mail: dr98@le.ac.uk

twitter: @damian_roland

\section{Abstract:}

The concept of early warning systems to detect deterioration in patients in hospitals is well established in health care systems. However their implementation has not always been in parallel with the available evidence. The face validity of tools that alert staff to early changes in physiological and observational parameters is a significant driver to their spread. However there are a number of factors which should give health care providers pause for thought. This review examines some common questions raised about PEWS (Paediatric Early Warning Systems) and discusses how we might use them to their maximum potential.

- First published in Paediatrics and Child Health (United Kingdom) 01 Jan 2017

http://www.paediatricsandchildhealthjournal.co.uk/article/S17517222(17)30006-9/abstract 


\section{Background}

There are some illnesses, injuries and disease processes that can't be altered and therefore, regardless of the best medical care, the outcome for some children and young people will always be tragic. However it is an uncomfortable paradigm that death may be an avoidable event in the very institution which should be expert at preventing it. Internationally, improvement bodies and regulators have raised concerns over preventable mortality in in-patients but while the chain of events needed to respond to the deteriorating child is clear; the best methodologies of doing this have yet to be clearly defined (Figure 1).

\section{Figure 1}

\section{Chain of events needed to demonstrate an improved response to deterioration in in-patient children}

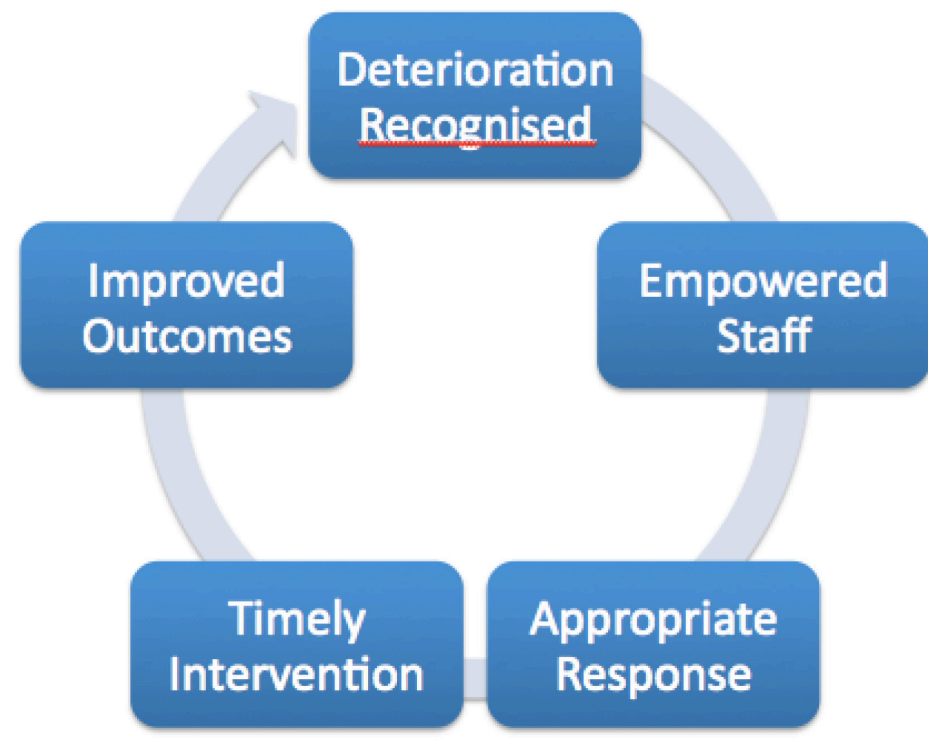

Ioffe AR, Anton NR, Burkholder SC. Reduction in Hospital Mortality Over Time in a Hospital Without a Pediatric Medical Emergency Team: Limitations of Before-and-After Study Designs.

Arch Pediatr Adolesc Med 2011;165(5):419-423.

\section{Scores vs Systems}

A number of systematic reviews have explored the range of tools developed in hospital deterioration but there is no one tool that appears to deliver the required specificity and sensitivity. This is a complex area of research as definitions are not universal and outcomes extremely variable. For example the term PEWS has been used to mean Paediatric Early Warning Score and Paediatric Early Warning System. A score being the amalgamation of various physiological and observational parameters into a singular descriptor of their extent of 
deviation from normal. Track and Trigger is a term used to describe this process with various models having been published (Table 1).

A system includes how the escalation and response to a change in score occurs. Self-evidently this needs to be robust if you are going to affect patient outcomes. Unfortunately the outcome you are looking to alter is often a challenge to define. Mortality is a rare event in children's hospitals, so much so that studies have struggled to run over long enough time periods to demonstrate significant change. Admission to intensive care is often used as a proxy but generates bias when comparing tertiary institutions (which contain their own units) with secondary care hospitals (which need to transfer patients to tertiary centres). Furthermore smaller hospitals are rarely able to provide a rapid response teams (a dedicated group of health care professionals who can be called if there are concerns about a child) in addition to the child's normal medical and nursing care.

\section{Defining Outcomes}

As a result of these, and other challenges, PEWS (scores and systems) have developed in a somewhat ad hoc fashion. In the United Kingdom there has been a clear growth in the use of PEWS but a huge amount of variation in their design and use. A number of potential challenges have surfaced which need to be addressed if we are to improve patient outcomes, one of which is having standard outcomes and definitions for illness.

Being able to recognise an ill child is a fundamental component of medical training. Everyone thinks they know what ill looks like. However although for individual diseases we have stratification of severity (e.g. asthma and croup scores) we rarely record a standard definition of illness which is linked to outcome. The Paediatric Index of mortality score score (PIM2) is one such system and provides a way of evaluating performance between different units. There is no similar ward based tool and the fact that PICU admission occurs on a relatively infrequent basis means PIM2, or equivalents, are probably not useful to apply on the wards. Location of care describes the differences in dependency between ward, high dependency and intensive care units as a measure of severity of illness regardless of underlying condition. However staffing, capacity and skill mix often mean that a child may be placed in a location which is not optimal or required for their needs. Standardisation of illness is relevant as it would help us understand in which situations a PEWS (system) approach would be beneficial and when the outcome of illness is unlikely to be altered. This is particularly important for non-ward based areas where children are seen. Of relevance are Emergency Departments where children are often untreated prior to arrival and may demonstrate extremely prompt deterioration. Numerous studies have failed to show an impact of PEWS in these environments. The unique physiology of children with cardiac lesions has prompted the development of cardiac specific PEWS. Similar arguments can be made for oncology and long term ventilation units. The heterogeneity of illness is one of the reasons why senior medical and nursing staff reject PEWS, "it will work on 
their ward, but not ours". This is compounded by the fact they have witnessed first hand the realities that dispel two common myths.

\section{Myth 1: Abnormal observations mean abnormal outcomes}

Because fever and distress can elevate heart and respiratory rate in the absence of serious bacterial illness it is challenging to create vital sign tables which do not trigger a large number of false positives. While an increasingly elevated heart rate is often noticed in retrospect following serious adverse events, tachycardia in children is common. In a study of children on a small hospitals observation ward 43\% (148/334) of those who were ultimately diagnosed has having minor illness (conditions in which the child would recover without treatment and without sequelae) had an elevated heart rate. An extremely large American study determined that 6122 of 40356 included patients met Systemic Inflammatory Response Syndrome criteria but 81.6\% (4993 patients) of these were discharged without treatment or return within 72 hours. The belief that observations generate additional work often reduces engagement (figure 2). The impact of this has been quantified in an emergency department where it was shown review activations may have generated an additional 7060 mins of work for no net benefit.

\section{Figure 2 A PEWS outcomes grid}

\section{PEWS Activates}

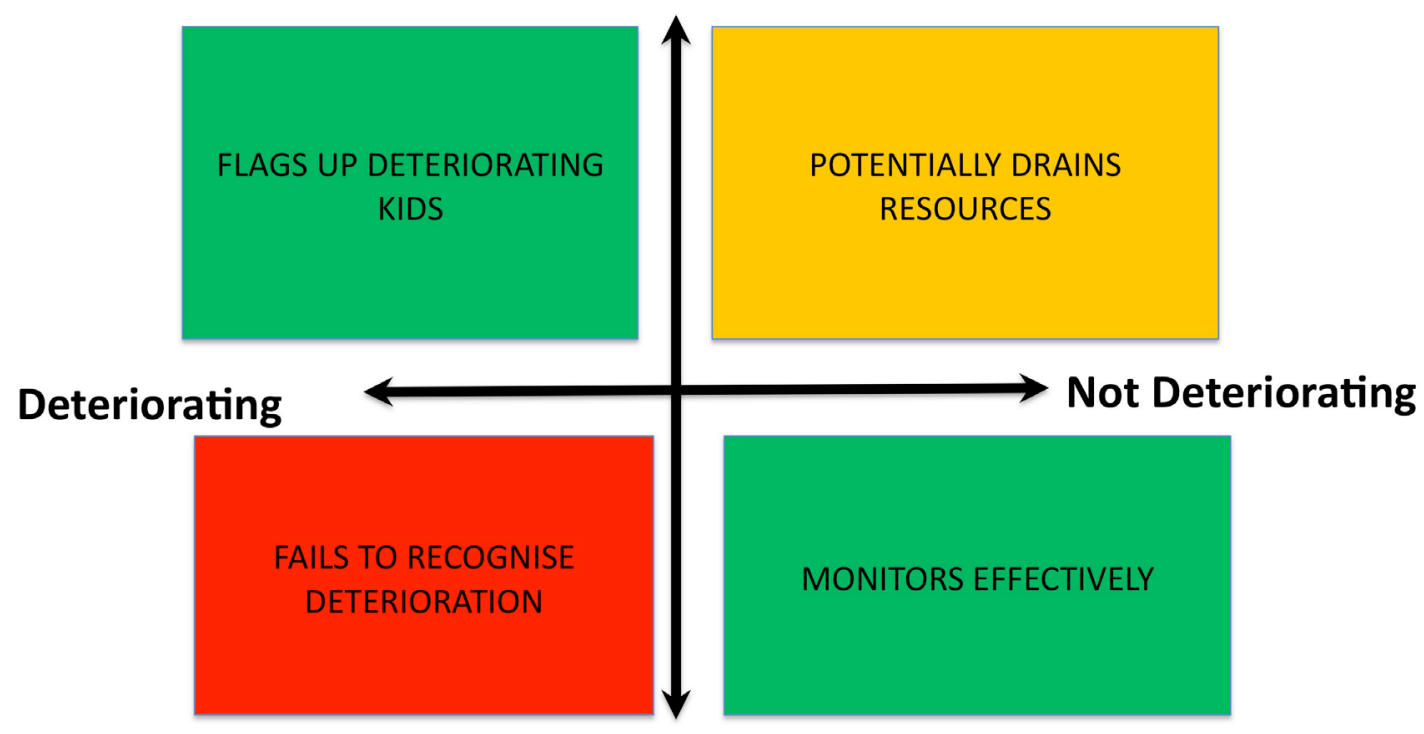

PEWS doesn't activate

Original concept by Akmal Hussein, Royal Berkshire Hospital

@damian_roland

Myth 2: Normal sets of observations mean normal outcomes 
Conversely it is not always the case the absence of deranged physiology is reassuring. In a study conducted in a large tertiary hospital in the UK there were 11 adverse outcomes in children with only $0-1$ sets of abnormal observations. To avoid missing the child who is unwell despite normal observations integration of clinician gut feeling, sometimes described as tacit knowledge, into PEWS occurs in a number of systems. However the individual impact of this is not clear. The concept of identifying 'watchers' is increasing in use. This approach allows staff to identify children on their wards who they feel are most at risk of deterioration. This is not just based on their clinical background but the instincts of staff who are caring for them. Parent involvement is also recognised at being invaluable in detecting the child who may not trigger on established scoring mechanisms but appear 'not quite right' to their carers. A study in America demonstrated that parent 'red' calls were more accurate than clinician ones.

\section{The Now}

Creating a PEWS (system) which is able to reliably recognise the deteriorating child, taking into account parental and clinician concerns, escalate appropriately and allow for an appropriate response is clearly a challenge. However ignoring this challenge is not an option. Currently there are a number of studies that might clarify the best approaches to some of these difficult questions. Parshurum et al.'s multi centre validation of BPEWS (bedside PEWS) entitled "Evaluating processes of care and outcomes of children in hospital (EPOCH)" is an international study using all cause mortality as a primary outcome [21] and results are due in 2016/17. The PUMA (Paediatric Early Warning System: utilization and mortality avoidance) study (www.pumastudy.com) has used a systematic review to introduce a track and trigger tool implementation package and aims to identify the contextual factors that are consequential for tool effectiveness, as well as highlight the key ingredients of successful implementation and normalisation. It is using a combination of quantitative and qualitiative methodologies.

While awaiting the outcome of these studies NHS Improvement in conjunction with the Royal College of Paediatrics and Child Health have produced a safe system framework for children at risk of deterioration. The system focuses on 4 groups:

1. Infants, children and young people, and their families

2. Clinicians and the wider team - this includes doctors, nurses, pathologists, pharmacists, radiologists, etc.

3. Local organisations and service providers

4. National organisations with leadership roles - such as NHS Improvement, NHS England, Royal College of Paediatrics and Child Health, Royal College of Nursing, etc.

These 4 groups run through 6 core elements (Table 2) demonstrating that engagement is needed across systems and organisations (Figure 3) 
The resource pack produced with the framework

(http://www.rcpch.ac.uk/safer-system-children-risk-deterioration) should help local health providers examine their own systems.

\section{The Future}

Technology is likely to play a role in the future of PEWS. It is known the design of observation charts makes a difference to their functionality and uptake. As whole hospital electronic records become more widespread attention to the look and feel of electronic observation on hand held and portable devices may become increasingly relevant. However use of devices as part of PEWS (system) may not produce the expected benefits. Alarm systems may be potentially significant cause of error due to alarm fatigue suggesting it is likely we can improve the methodology of specifically highlighting the vital signs that need review. Finally the vast amount of data that can be collected and analysed at pace means PEWS can be evaluated in real time and on an iterative basis. Updates and alterations to scores or responses may be targeted to specific seasons, hospital flow and acuity.

\section{Figure 3}

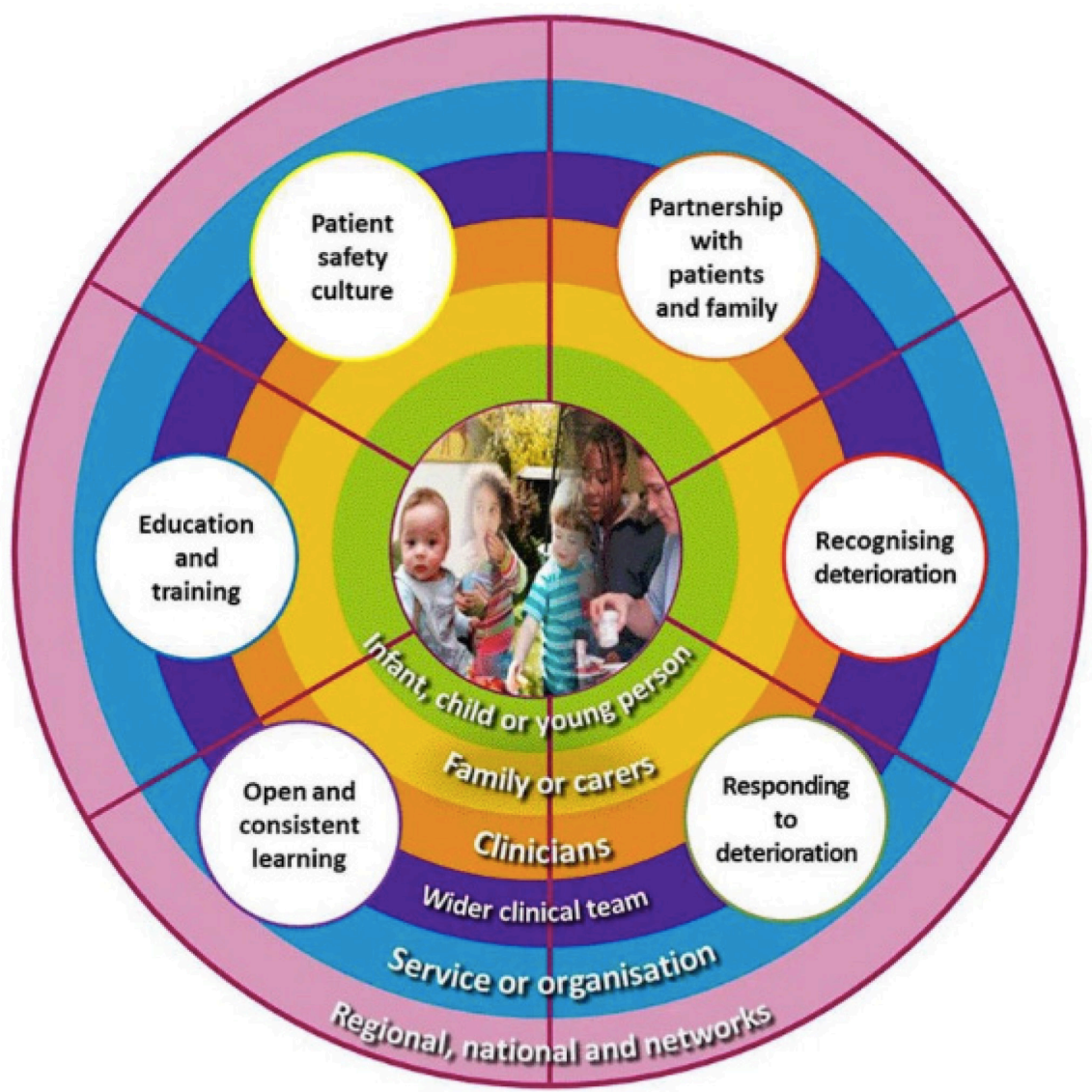

\section{Conclusion}


There is no magic bullet that is going to ameliorate the problem of reliably detecting the deteriorating child. However new evidence is being produced at a rapid pace and an increasing understanding of the chain of events necessary to improve outcomes will help providers find interventions for their particular environments.

\section{Acknowledgements}

The hard work and dedication of Jayne Wheway in developing the Safe System Framework on behalf of NHS Improvement and the Royal College of Paediatrics and Child Health

\section{Competing Interests}

Dr. Roland is co-investigator on the PUMA study and helped design the Safe System Framework

\section{Further Reading}

[1] Roland D But I told you she was ill. The role of families in preventing avoidable harm in children BMJ Qual Saf 2015;24:186-187

[2] Chapman SM, Wray J, Oulton K and Peters MJ. Systematic review of paediatric track and trigger systems for hospitalised children. Resuscitation. 2016 Aug 3 doi: 10.1016/j.resuscitation.2016.07.230. [Epub ahead of print]

[3] NICE. Acutely Ill Patients in Hospital: Recognition and Response to Acute Illness in Adults in Hospitals, 2007.

[4] Roland D, Oliver A, Edwards E, et al . Use of paediatric early warning systems in Great Britain: has there been a change of practice in the last 7 years? Arch Dis Child 2014;99:26-9.

[4] Challen K and Roland D. Early Warning Scores: a health warning EMJ 2015 doi:10.1136/emermed-2014-204250

[6] Roland D, McCaffery K, and Davies F. (2016) Scoring systems in paediatric emergency care: Panacea or paper exercise? Journal of Paediatrics and Child Health 52 (2), 181-186.

[7] McLellan MC, Gauvreau K, Connor JA Validation of the Cardiac Children's Hospital Early Warning Score: an early warning scoring tool to prevent cardiopulmonary arrests in children with heart disease. Congenit Heart Dis. 2014 May-Jun;9(3):194-202

[8] Thompson M, Coad N, Harnden A, Mayon-White R, Perera R, Mant D How well do vital signs identify children with serious infections in paediatric emergency care Arch Dis Child 2009;94:888-893

[9] Scott HF, Deakyne SJ, Woods JM, Bajaj L. The prevalence and diagnostic utility of systemic inflammatory response syndrome vital signs in a pediatric emergency department. Acad Emerg Med. 2015 Apr;22(4):381-9.

[10] O'Leary F and Chayan G. Predicting the impact on workload with the application of inpatient clinical review criteria into a paediatric emergency department. Emergency Medicine Australasia (2011) 23, 748-753

[11] Edwards E, Powell C,Mason B,Oliver A Prospective cohort study to test the 
predictability of the Cardiff and Vale paediatric early warning system Arch Dis Child 2009;94:602-606

[12] Brady PW, Zix J, Brilli R, et al. Developing and evaluating the success of a family activated medical emergency team: a quality improvement report. BMJ Qual Saf 2015;24: 203-11.

[13] Parshuram CS, Dryden-Palmer K, Farrell C Evaluating processes of care and outcomes of children in hospital (EPOCH): study protocol for a randomized controlled trial. Trials. 2015 Jun 2;16:245.

[14] Christofidisa M,Hill A, Horswill M, Watsona M A human factors approach to observation chart design can trump health professional prior chart experience Resuscitation 84 (2013) 657- 66

[15] Bonafide CP, Lin R, Zander M, et al. Association between exposure to nonactionable physiologic monitor alarms and response time in a children's hospital. J HospMed. 2015;10(6):345-351.

[16] Bonafide C, Roland D and Brady P. Rapid Response Systems 20 Years Later New Approaches, Old Challenges. JAMA Paediatrics Published online June 20, 2016. doi:10.1001/jamapediatrics.2016.0398 
Table 1 - Definitions of Track and Trigger Systems

\begin{tabular}{|c|c|}
\hline \multicolumn{2}{|c|}{$\begin{array}{l}\text { Four Categories of Track and Trigger System } \\
\text { (as defined by the National Institute for Clinical Excellence, NICE) }\end{array}$} \\
\hline Single Parameter System & $\begin{array}{l}\text { Periodic observation of selected vital signs that } \\
\text { are compared with a simple set of criteria with } \\
\text { predefined thresholds, with a response algorithm } \\
\text { being activated when any criterion is met. }\end{array}$ \\
\hline Multiple Parameter System & $\begin{array}{l}\text { Response algorithm requires more than one } \\
\text { criterion to be met, or differs according to the } \\
\text { number of criteria met. }\end{array}$ \\
\hline Aggregate Scoring System & $\begin{array}{l}\text { Weighted scores are assigned to physiological } \\
\text { values and compared with predefined trigger } \\
\text { thresholds. }\end{array}$ \\
\hline Combination System & $\begin{array}{l}\text { Single or multiple parameter systems used in } \\
\text { combination with aggregate weighted scoring } \\
\text { systems. }\end{array}$ \\
\hline
\end{tabular}


Table 2 - Core Elements of the Safe System Framework

\begin{tabular}{|c|c|}
\hline Patient Safety Culture & $\begin{array}{l}\text { A large and challenging element covering } \\
\text { many aspects that all groups are now } \\
\text { trying to define and develop, including a } \\
\text { commitment to overall improvement in } \\
\text { patient safety, prioritising safety, } \\
\text { leadership and executive accountability, } \\
\text { and monitoring and measuring patient } \\
\text { safety }\end{array}$ \\
\hline $\begin{array}{l}\text { Partnerships with Patients and } \\
\text { Families }\end{array}$ & $\begin{array}{l}\text { While all of the core elements focus on the } \\
\text { patient and family, this partnership is an } \\
\text { area of increased growth and central to } \\
\text { supporting all the others }\end{array}$ \\
\hline Recognising Deterioration & $\begin{array}{l}\text { The ability to spot physiological deviations } \\
\text { before significant changes in care are } \\
\text { needed or harm occurs is a fundamental } \\
\text { working element which is central to the } \\
\text { system }\end{array}$ \\
\hline Responding to Deterioration & $\begin{array}{l}\text { Ensuring a timely and accurate response } \\
\text { encompassing all necessary support and } \\
\text { treatment from all those involved in the } \\
\text { care of the patient is the vital element that }\end{array}$ \\
\hline
\end{tabular}




\begin{tabular}{|l|l|}
\hline Open and Consistent Learning & is often the key change required \\
& $\begin{array}{l}\text { Consideration of the system errors and } \\
\text { investigating and evaluating incidents as } \\
\text { well as best practice in order to learn and } \\
\text { effect change will drive forward continual } \\
\text { improvements in all elements }\end{array}$ \\
\hline Education and Training & $\begin{array}{l}\text { Consistently building clinical knowledge } \\
\text { and capability as well as patient safety and } \\
\text { improvement methods will provide the } \\
\text { foundation for all elements to be enhanced }\end{array}$ \\
\hline
\end{tabular}

